\title{
Endodoncia guiada Para el manejo de Canales Obliterados, Reporte de Caso
}

\section{Guided Endodontic Treatment for the Management of Obliterated Pulp Canals. A Case Report}

\author{
Jaime Abarca ${ }^{*}$, Constanza Barraza ${ }^{1}$, Pablo Matamala ${ }^{2,3}$, Gustavo Mazzey $^{4}$, Héctor Monardes ${ }^{1}$
}

1.Postítulo Especialización Endodoncia, Facultad Odontología Universidad San Sebastián. Santiago, Chile

2.Hospital del Salvador, Providencia, Chile

3. CRS Cordillera Oriente, Peñalolén, Chile

4.Postítulo Especialización Implantología,

Universidad San Sebastián.

* Correspondencia Autor: Jaime Abarca Reveco | Dirección: Lago Panguipulli 1390, Puerto Montt, Chile. | Teléfono: +569 77979398 | E-mail: jaime. abarca@uss.cl

Trabajo recibido el 03/08/2020.

Trabajo revisado 11/01/2021

Aprobado para su publicación el 15/02/2021

\begin{abstract}
RESUMEN
El objetivo de este reporte es presentar el tratamiento endodóntico de un diente anterior con obliteración del canal radicular, con la ayuda de la endodoncia guiada. Paciente masculino sin antecedentes médicos relevante, y en tratamiento ortodóntico. Diente 2.1 con historia de traumatismo dento-alveaolar y presencia de dolor a la percusión. En la TCHC se observa obliteración total del canal radicular en el tercio cervical y medio y un área de radiolucidez apical. Diagnóstico: Periodontitis apical Sintomática. Tratamiento: Terapia de canal No vital, con planificación virtual y guía endodóntica. Se utilizó el programa de acceso gratuito, Bluesky Plan $^{\circledR}$, para la planifica-ción digital de la guía endodóntica. El tratamiento se realizó en dos citas. Conclusión: el uso de tecnología para la resolución de casos de alta complejidad en endodoncia, podría ayudar a mejorar el pronóstico de estos dientes y ser una alternativa más segura para reducir la posibilidad de perforación y preservar estructura dental.
\end{abstract}

PALABRAS CLAVE:

Obliteración del canal radicular; Periodontitis apical; Tomografía computacional de haz cónico.

Int. J. Inter. Dent Vol. 14(2); 187-190, 2021.

\section{ABSTRACT}

The aim of this case report is to present the endodontic treatment of an anterior tooth with an obliterated pulp canal, with the help of guided endodontics. Male patient with no relevant medical history, currently with orthodontic treatment. Tooth 2.1 had a history of dental trauma and presented tenderness to percussion. The CBCT exam showed a completely obliterated root canal in the cervical and middle third, and apical radiolucency. Diagnosis: Symptomatic Apical Periodontitis. Treatment: Root canal treatment with virtual planning and endodontic guide. Bluesky Plan® free access program was used for the digital planning of the endodontic guide. The treatment was made in two sessions. Conclusion: the use of technology for the resolution of high complexity cases in endodontics could help improve the prognosis of these teeth and be a safer alternative to reduce the possibility of perforation and preserve more tooth structure.

\section{KEY WORDS:}

Apical periodontitis; Cone-beam computed tomography; Pulp canal obliteration.

Int. J. Inter. Dent Vol. 14(2); 187-190, 2021.

\section{INTRODUCCIÓN}

La obliteración del canal radicular, también llamada metamorfosis cálcica (MC), es entre otras causas, una secuela del traumatismo dentoalveolar y se desarrolla con mayor frecuencia en los dientes posterior a una concusión y subluxación ${ }^{(1)}$. Se caracteriza por la deposición de tejido duro en el espacio del canal radicular y presentar cambio de coloración amarillento, disminución o falta de sensibilidad afectando los dientes anteriores de pacientes adultos jóvenes ${ }^{(2)}$. Sin embargo, el riesgo de necrosis, incluso en el caso de restauraciones, prótesis o tratamiento de ortodoncia es limitado(3).

El tratamiento endodóntico es recomendado solo cuando se presentan signos o síntomas de periodontitis apical(4). La Asociación Americana de Endodoncia considera el tratamiento de dientes calcificados como de alto nivel de dificultad ${ }^{(5)}$.

Históricamente los odontólogos han localizado los canales de manera táctil, sin embargo el desarrollo de instrumentos ha ayudado a mejorar esta modalidad de tratamiento. La magnificación mejora la visibilidad de la cavidad pulpar y algunas herramientas auxiliares, como la micro sonda DG16 y las puntas ultrasónicas, facilitan la búsqueda del orificio del canal radicular utilizando el microscopio(6).

Incluso con el uso de un microscopio dental, la preparación de una cavidad de acceso puede conducir a una pérdida excesiva de estructura dental lo que reduce el pronóstico a largo plazo del diente ${ }^{(5)}$.

Recientemente, se ha desarrollado un nuevo enfoque de tratamiento para los dientes con obliteración del canal radicular mediante guías, confeccionadas digitalmente, que direccionan la fresa hacia el canal radicular calcificado ${ }^{(5)}$.

El presente reporte describe el tratamiento endodóntico realizado en un diente con el canal radicular obliterado en un paciente con tratamiento de ortodoncia y con antecedentes de trauma de larga data, con la ayuda 
de las imágenes de Tomografía Computarizada de Haz Cónico (TCHC) y guía endodóntica 3D confeccionada a través de una planificación virtual.

\section{REPORTE CASO}

Paciente de género masculino de 28 años, sin antecedentes médicos relevantes, Clase esqueletal tipo III, en tratamiento ortodóntico pre quirúrgico, es referido al Programa de Especialización en Endodoncia de la Universidad San Sebastián para evaluación y posibilidad tratamiento del diente 2.1 con historia de traumatismo dento-alvealor antiguo.

Al examen clínico, el paciente presenta aparatología fija en maxilar y mandíbula, cambio de coloración amarillento en corona clínica de diente 2.1. (Figura 1) Sin respuesta frente a las pruebas de sensibilidad térmicas y a la estimulación eléctrica, y una respuesta positiva a la percusión.

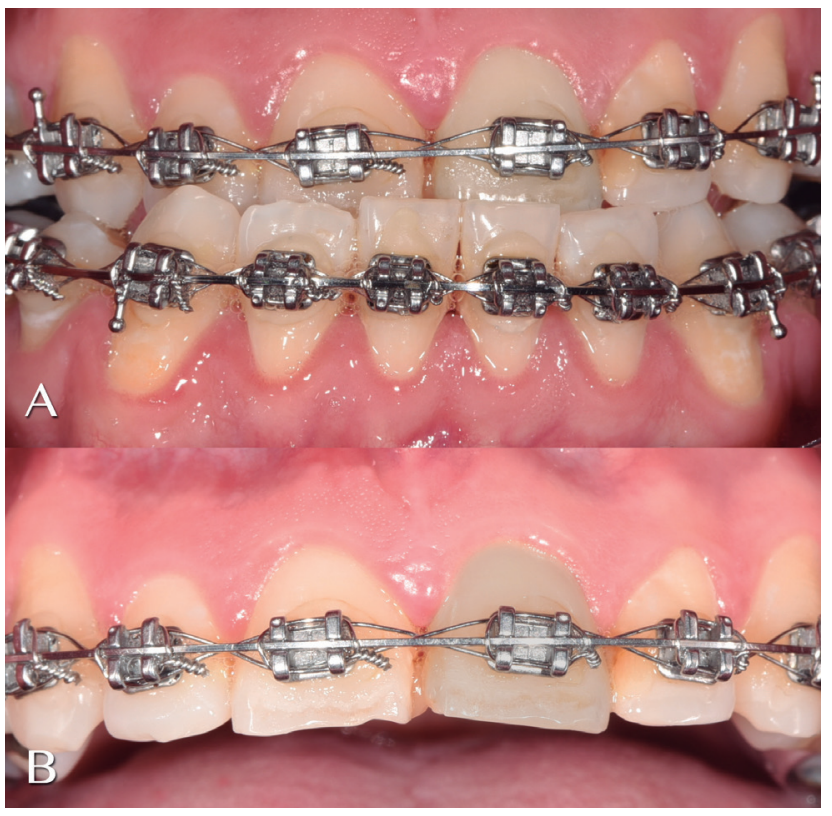

Figura 1. Imagen clínica inicial, se observa el cambio de coloración diente 2.1

Al examen radiográfico retroalveolar se observa diente 2.1 con obliteración total del canal radicular y lesión apical. En la TCHC se observa canal radicular totalmente obliterado en tercio cervical y medio, solamente visible en 1/3 apical y claros signos de Periodontitis Apical. (Figura 2)

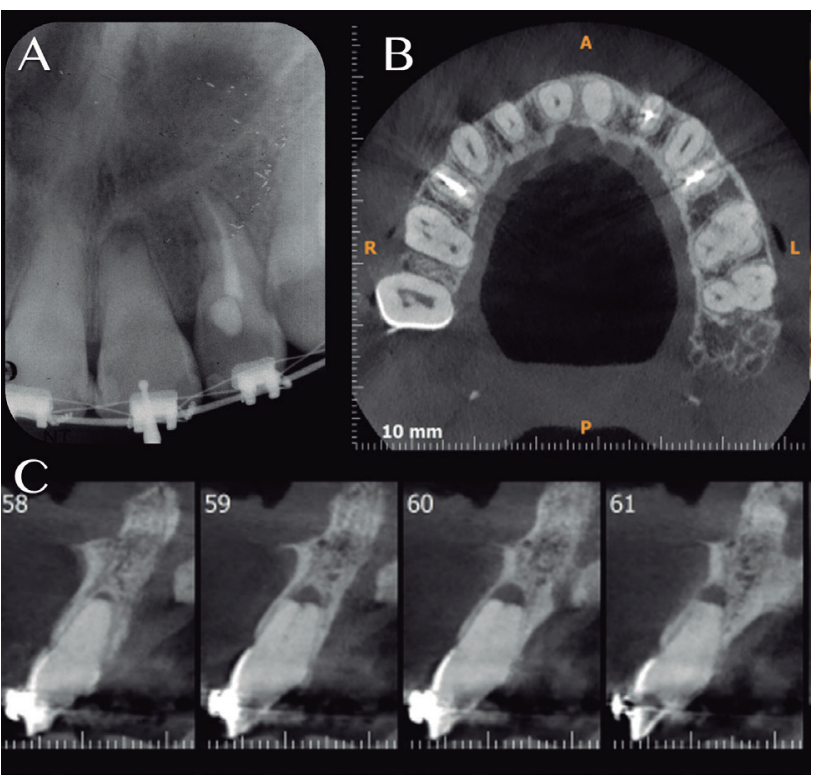

Figura 2. A Radiografía Inicial, B Vision axial TCHC y C Vison Sagital TCHC.
Diagnóstico: Se realizan pruebas diagnósticas diferenciales entre periodontitis apical y Rompimiento Apical Transitorio (TAB por sus siglas en inglés), debido a la historia de trauma y al tratamiento de ortodoncia, pues esta condición también se puede asociar a deposición de dentina dentro del canal radicular. Se diagnostica Periodontitis Apical Sintomática, en diente con Obliteración Radicular, pues el trauma que sufrió el diente es de muy larga data, el cambio de color de la corona se ha mantenido en el tiempo y los tejidos periapicales no se recuperaron, además de la persistencia en el tiempo de dolor a la percusión y de la ausencia permanente de sensibilidad frente a los cambios de temperatura.

Tratamiento, Se indicó tratamiento de canal radicular, debido a la permanente sensación de dolor a la percusión por años, y a la evidente falta de reparación de los tejidos periapicales, con planificación virtual y guía endodóntica 3D, por la ausencia de canal radicular visible en los exámenes radiográficos.

Previa solicitud y firma de consentimiento informado por parte del paciente, en una primera visita se realiza impresión maxilar con silicona por adición STD ${ }^{\mathrm{TM}}(3 \mathrm{M})$ y se confecciona modelo para realizar impresión digital y obtención de archivos STL. Para la planificación digital de la guía endodóntica se utilizó el programa de libre acceso Bluesky Plan ${ }^{\circledR}$, donde se realizó la fusión Dicom-Stl del caso, para luego planificar una guía de acceso. La lógica de tratamiento fue la colocación de un implante personalizado de $15 \mathrm{~mm}$ de largo con diámetro oclusal $2 \mathrm{~mm}$ y diámetro apical de $1 \mathrm{~mm}$, la posición del implante coincidía con el recorrido del canal calcificado, y terminaba en el tercio apical donde se podía observar el canal en la TCHC

Con el software Bluesky Plan $^{\circledR}$ se generó una guía quirúrgica $100 \%$ digital donde se dejó un agujero guía de $1,69 \mathrm{~mm}$ de diámetro para el paso de fresa, $100 \mu$ mas gran-de que el tamaño del vástago de una fresa estándar de alta velocidad. Para lograr una mayor estabilidad de la guía, el diseño de la guía fue de un primer molar a otro. Obtenido el archivo digital la guía fue prototipada en una impresora 3D Wanhao Duplicator 7 de tecnología DLP-LCD con resina estándar color negro. (Figura 3). Cabe desatacar que la aparotología fija presente era un desafío para el ajuste y estabilidad de la guía de acceso endodóntico, por lo que el diseño de esta debió abarcar todas las superficies dentarias que estuvieran libres.

En una segunda cita se procede al tratamiento endodóntico utilizando en el acceso la guía endodóntica 3D y fresa de tallo largo $25 \mathrm{~mm}$ de largo por $0,12 \mathrm{~mm}$ de diámetro (Jota), hasta localizar el canal (Figura 4), posteriormente se realiza aislamiento absoluto, (Figura 5) Se continúa con la preparación del canal con sistema Reciproc ${ }^{\circledR}$ R50 VDW, irrigación final con hipoclorito de sodio 5\% y EDTA 17\% mas activación sónica, obturación del 1/3 apical con Biodentine ${ }^{\circledR}$ (Septodont), debido a la presencia de reabsorción apical, y el resto del canal se obturó con inyección de gutapercha termoplastificada con sistema Calamus Dual ${ }^{\circledR}$ (Dentsplay-Sirona) y cemento sellador TopSeal ${ }^{\circledR}$ (Dentsplay-Sirona). El sellado coronal de la cavidad de acceso se realizó con Resina A3 Z350 ${ }^{\circledR}$ (3M).

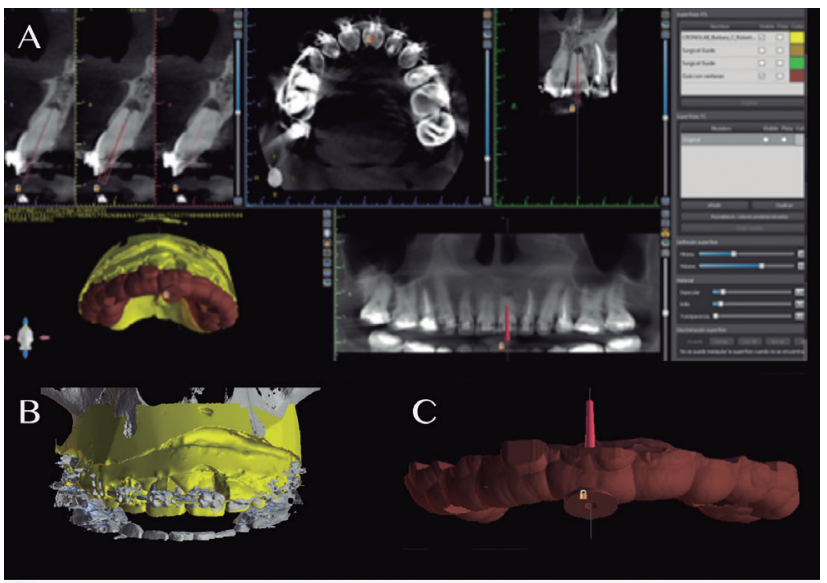

D

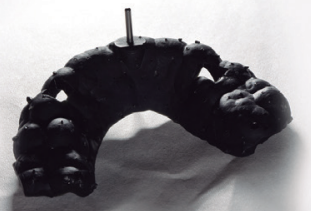

Figura 3. A Planificación Virtual en Bluesky, B Fusión digital de archivos STL y DICOM en Bluesky, C Guía Endodóntica digital y D Guía endodóntica en resina y fresa de tallo largo. 

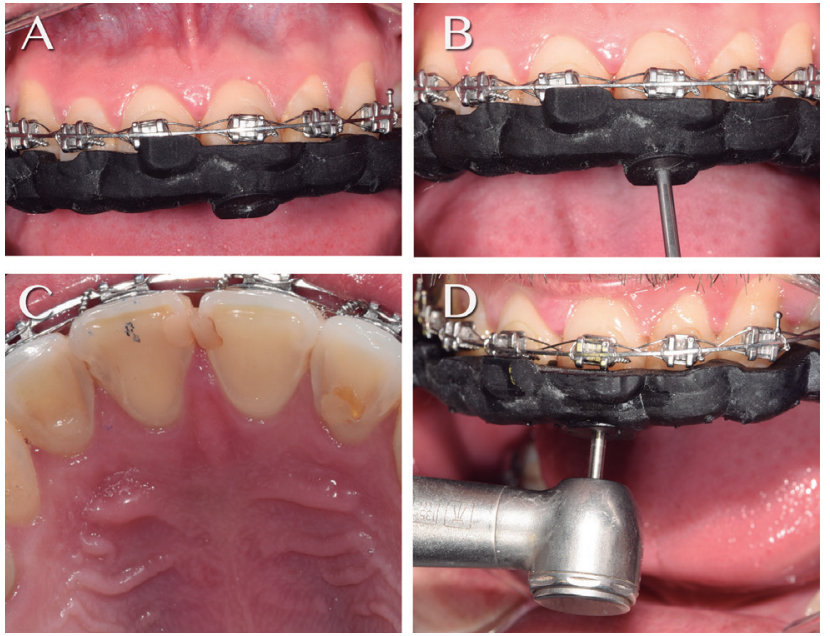

Figura 4. A Prueba de estabilidad de guía, $B$ prueba de fresa tallo largo, $C$ Zona de acceso de fresa y $D$ Acceso realizado.

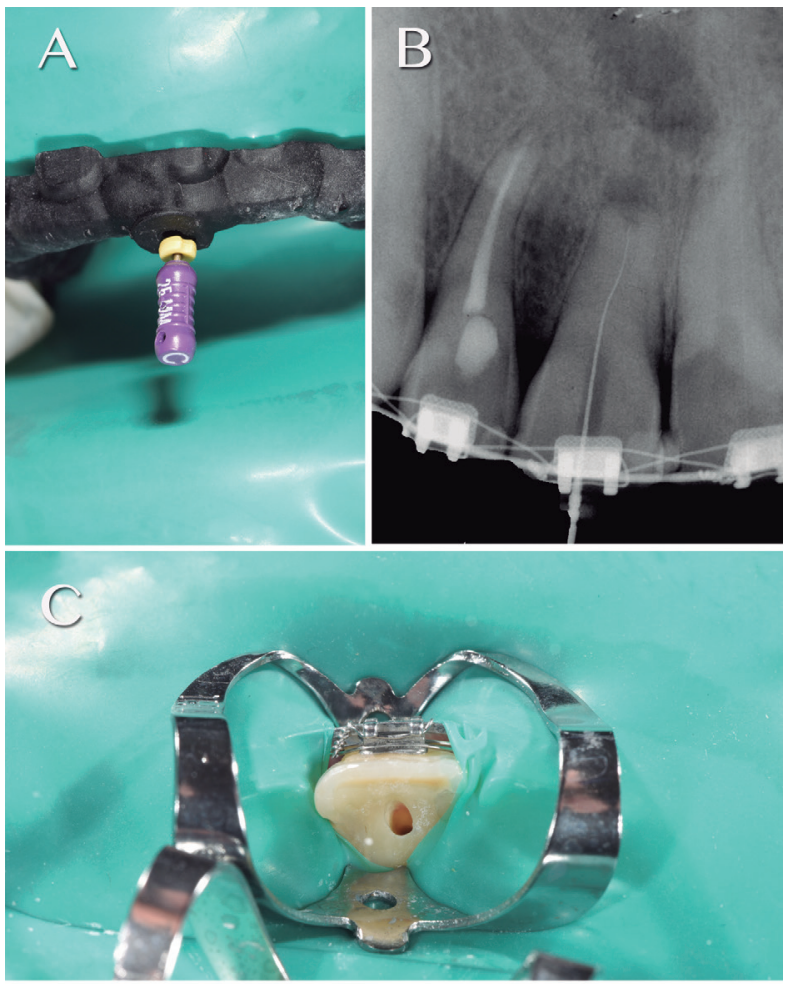

Figura 5. A Localización canal con la guía instalada, B Rx determinación longitud de trabajo y $\mathrm{C}$ imagen clínica acceso conservador.

El paciente acude a control programado a los 12 meses, ya con la cirugía ortognática realizada, con signos clínicos y radiográficos de reparación. (Figura 6), lo que permite asegurar que el diagnóstico y el tratamiento realizado han sido los correctos y se puede formular un pronóstico favorable del diente, tanto por los resultados clínicos como por los cambios observados en la radiografía.

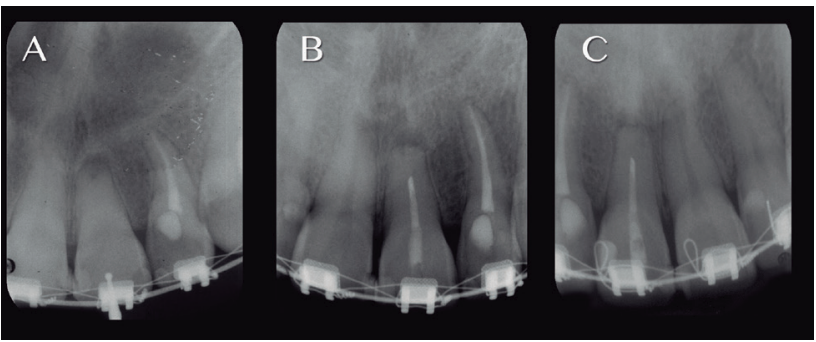

Figura 6. A Rx previa, B Rx control obturación, 1/3 apical sellado con Biodentine, radiopacidad similar a dentina y C Rx control 12 meses.

\section{DISCUSIÓN}

La obliteración del canal radicular se considera un signo de vitalidad pulpar y, a menos que exista evidencia clínica y radiográfica de necrosis pulpar, no está indicado el tratamiento del canal radicular. Sin embargo, hasta un tercio de los dientes con obliteración del canal radicular pueden desarrollar patología apical a largo plazo ${ }^{(7,11)}$

La obliteración del canal radicular en casos de periodontitis apical es un desafío, aunque endodoncistas con experiencia tienen altos niveles de éxito, en estos casos los riesgos de perforación e incluso pérdida del diente deben ser considerados ${ }^{(8)}$.

En al año 2015 la Asociación Americana de Endodoncia y la Academia Americana de Radiología Oral y Máxilo Facial recomienda el uso de TCHC para la localización de canales calcificados por su alto grado de dificultad ${ }^{(5)}$. En casos de dientes con obliteración del canal radicular, incluso con el uso de un microscopio dental, la preparación de una cavidad de acceso puede conducir a una pérdida excesiva de sustancia que deteriora la estabilidad y, por lo tanto, reduce el pronóstico a largo plazo del diente ${ }^{(5)}$.

Este reporte describe el tratamiento a través de una Guía Endodóntica 3D que podría hacer más predecible al tratamiento de dientes con obliteración del canal radicular y periodontitis apical evitando la pérdida excesiva de tejido dentinario en la preparación del acceso, al realizar una cavidad mínimamente invasiva, que reduce el riesgo de fractura comparado con los accesos tradicionales ${ }^{(9)}$. Además, con este método se disminuyen las posibilidades de realizar perforaciones, se reduce el tiempo de trabajo y puede ser realizado por operadores con menos experiencia, incluso obviando el microscopio ${ }^{(4,8)}$

En investigaciones ex vivo, diversos autores evaluaron las preparaciones de acceso guiado mediante la superposición de un $\mathrm{TCHC}$ inicial y posterior al acceso ${ }^{(5,10,11)}$. Buchgreitz et al. ${ }^{(10)}$ encontraron que la desviación media de las cavidades de acceso es inferior al umbral de $0,7 \mathrm{~mm}$ definido por el radio de la fresa más el radio del canal radicular. Zehnder et al.(5) y Connert et al.(11) también encontraron pequeñas desviacio-nes del acceso previsto $(0,12$ a $0,34 \mathrm{~mm}$ en la punta de la fresa) y una desviación angular media de menos 2 grados. Connert et al.(12) compararon la capacidad de localización del canal, la cantidad de estructura dentaria perdida y el tiempo requerido para realizar el acceso entre la técnica convencional y el uso de guías endodónticas, encontraron que el éxito en la localización fue de $41,7 \%$ versus $91,7 \%$ que la media de estructura perdida fue $49,9 \mathrm{~mm}^{3}$ y $9,8 \mathrm{~mm}^{3}$ y el tiempo utilizado fue de 21.8 minutos versus 11.3 minutos, respectivamente. Además, encontraron que el éxito del acceso guiado no está relacionado con la experiencia del operador. Estas investigaciones sugieren que las guías de acceso impresas en 3D representan un medio eficiente y seguro para abordar los desafiantes escenarios endodónticos, permitiendo tanto el desbridamiento químico-mecánico como la conservación de la estructura dental.

La cirugía apical es un enfoque más invasivo e incómodo para el paciente; sin embargo, debe ser considerado como el tratamiento de elección en casos de obliteración del canal radicular en que una línea recta al canal no se puede lograr con la fresa en la endodoncia guiada, como en los casos de canales con curva severas ${ }^{(8)}$

\section{CONCLUSIÓN}

El uso de tecnología para la resolución de casos de alta complejidad en endodoncia, como lo son la obliteración del canal radicular en diente con periodontitis apical, ayuda a mejorar el pronóstico de estos dientes y podría ser una alternativa más segura al disminuir la posibilidad de perforación y conservar más estructura dentaria.

\section{RELEVANCIA CLÍNICA.}

El uso de la tecnología y la planificación digital en endodoncia podría ser una alternativa para disminuir la posibilidad de perforación y conservar más estructura dentaria en el manejo de dientes con obliteración del canal radicular que requieran terapia de canales radiculares.

\section{CONFLICTO DE INTERESES}

Los autores declaran no presentar conflicto de interés.

\section{FUENTES DE FINANCIAMIENTO}

Este reporte no cuenta con financiamiento alguno. 


\section{Bibliografía}

1. McCabe PS, Dummer PM. Pulp canal obliteration: an endodontic diagnosis and treatment challenge. Int Endod J. 2012;45(2):177-97.

2. Abd-Elmeguid A, EISalhy M, Yu DC. Pulp canal obliteration after replantation of avulsed immature teeth: a systematic review. Dent Traumatol. 2015;31(6):437-41.

3. Andreasen FM, Kahler B. Pulpal response after acute dental injury in the permanent dentition: clinical implications-a review. J Endod. 2015;41(3):299-308.

4. Lara-Mendes STD, Barbosa CDM, Santa-Rosa CC, Machado VC. Guided endodontic access in maxillary molars using cone-beam computed tomography and computer-aided Design/Computer-aided manufacturing system: A Case Report. J Endod. 2018;44(5):875-9.

5. Connert T, Zehnder MS, Weiger R, Kuhl S, Krastl G. Microguided endodontics: accuracy of a miniaturized technique for apically extended access cavity preparation in anterior teeth. J Endod. 2017;43(5):787-90.

6. Shi X, Zhao S, Wang W, Jiang Q, Yang X. Novel navigation technique for the endodontic treatment of a molar with pulp canal calcification and apical pathology. Aust Endod J. 2018;44(1):66-70.

7. Oginni AO, Adekoya-Sofowora CA, Kolawole KA. Evaluation of radiographs, clinical signs and symptoms associated with pulp canal obliteration: an aid to treatment decision. Dent Traumatol. 2009;25(6):620-5.

8. Fonseca Tavares WL, Diniz Viana AC, de Carvalho Machado V, Feitosa Henriques LC, Ribeiro Sobrinho AP. Guided endodontic access of calcified anterior teeth. J Endod. 2018;44(7):1195-9.

9. Plotino G, Grande NM, Isufi A, loppolo P, Pedulla E, Bedini R, et al. Fracture strength of endodontically treated teeth with different access cavity designs. J Endod. 2017;43(6):995-1000.

10. Buchgreitz J, Buchgreitz M, Mortensen D, Bjorndal L. Guided access cavity preparation using cone-beam computed tomography and optical surface scans - an ex vivo study. Int Endod J.2016;49(8):790-5

11. Zehnder MS, Connert T, Weiger R, Krastl G, Kuhl S. Guided endodontics: accuracy of a novel method for guided access cavity preparation and root canal location. Int Endod J. 2016;49(10):966-72.

12. Connert T, Krug R, Eggmann F, Emsermann I, ElAyouti A, Weiger R, et al. Guided endodontics versus conventional access cavity preparation: a comparative study on substance loss using 3-dimensional-printed teeth. J Endod. 2019;45(3):327-31. 